\title{
Evaluation of the True View PCD Video Laryngoscope for Oral Endotracheal Intubation
}

\author{
Neha Agrawal ${ }^{\circledR 1}$, Alks Shah ${ }^{\circledR 2}$, Balraj Joshi ${ }^{\circledR 3}$, Pinal Vasani ${ }^{\circledR 4}$ \\ ${ }^{1}$ Department of Anaesthesia, GMERS Medical College Sola, Ahmedabad, Gujarat, India, ${ }^{2}$ Associate Professor, Department of Anaesthesia, GMERS Medical College Sola, \\ Ahmedabad, Gujarat, India, ${ }^{3} 3$ rd year resident, Department of Anaesthesia, GMERS Medical College Sola, Ahmedabad, Gujarat, India, ${ }^{4}$ Resident, Department of \\ Anaesthesia, GMERS Medical College Sola, Ahmedabad, Gujarat, India.
}

\section{Abstract}

Background: Truview PCD video laryngoscope is particularly planned to assist in locating the endotracheal tube in addition to observe the admission of the tube into the glottis. The present study was performed to assess the outlook of glottic opening and relief of intubation between the Truview PCD laryngoscope and Macintosh laryngoscope in patients undergoes general anaesthesia. Subjects and Methods: Ninety patients of ASA grade 1 and 2 aged 18-60 years, posted for elective surgery under general anaesthesia needing endotracheal intubation were arbitrarily allocated into group 1 (Truview PCD laryngoscope $n=45$ ) and group 2 (Macintosh laryngoscope $n=45$ ). The two groups were compared for demographic data, intubation difficulty score, Cormack-Lehane grade, time to intubate, number of intubation attempts and hemodynamic parameters. Conclusion: Truview PCD can be measured as an alternate intubation device, especially in difficult intubation conditions.

Keywords: Truview PCD video laryngoscope Mc cintosh Laryngoscope, tracheal intubation

Corresponding Author: Alks Shah, Associate Professor, Department of Anaesthesia, GMERS Medical College Sola, Ahmedabad, Gujarat, India.

E-mail: dralkap@yahoo.co.in

Received: 05 July 2020

\section{Introduction}

The anesthesiologist is the person solely responsible for airway management of the patient undergoing a surgical procedure. The difficulty may be encountered in mask ventilation, laryngoscopy, or intubation and often arises unexpectedly. ${ }^{[1]}$ The incidence of failed intubation from previous reports ranges between one in two hundred and fifty to one in seven hundred and fifty. Anaesthesia with a complicated airway in patients guides to straight airway trauma and morbidity from hypoxia and hypercarbia. ${ }^{[2,3]}$ Managing difficult airway sporadically keeps the augmented function of physical potency to the airway of the patient than is ordinary, which can cause straight airway trauma and disruption of gas exchange, which could ground brain damage and cardiovascular activation or depression. Straight mediated reflexes laryngovagal and laryngospinal offer the ultimate foundation of morbidity. Problems in managing "difficult intubation". may also arise in the peripheral hospitals where other aids to intubation in the form of laryngeal mask airways, lighted stylets, bougie, or fibreoptic bronchoscope may not be available, or the expertise to use these aids may be lacking. Endotracheal intubation in an unanticipated difficult airway situation can quickly turn into a matter of life and death. ${ }^{[4]}$ In this scenario any device, which can aid successful intubation is a boon to the anesthesiologist. All anaesthesiologists should be skilled in at least one alternative device and technique of tracheal intubation under vision. They include:

a) McCoy blade laryngoscope

b) Truview PCD laryngoscope

c) Airtraq

d) Combitube

e) Fibreoptic bronchoscope

Out of the above Truview PCD laryngoscope is a simple and relatively cheaper device that promises to be an alternative to Macintosh laryngoscope. Hence present research was conducted to assess the ease of intubation with help of a truview PCD laryngoscope. 


\section{Subjects and Methods}

Present research with 90 patients aged 18 to 60 years with ASA grade 1 and 2, either sex, planned to undertake possible surgery under general anaesthesia needing endotracheal intubation were incorporated in the study and assess preoperatively for difficult intubation parameters by an unbiased anaesthesiologist was unacquainted of the patient group allotment. This included: 1. Mouth opening (normal $>3 \mathrm{~cm}$ or restricted $<3 \mathrm{~cm}$ ), 2. Mallampati oropharyngeal view as per changed scoring of Samsoon and Young, 3. Thyromental distance 4. Temporomandibular joint (TMJ) mobility 5 . Neck movements, 6 . Condition of the teeth 7. Mandibular size (normal or micrognathia)

The anaesthesiologist was requested to provide a YES /NO reply to whether the intubation was tricky. Allocation of the selected patient to either Group 1 (Truview PCD laryngoscope) or Group 2 (Macintosh laryngoscope) was done as per the randomization service. The following patients were excluded from the study: Patients with a recognized history of sensitivity and contraindications to drugs utilized, a record of significant cardiac, respiratory, renal, hepatic or central nervous system diseases (ASA 3 and above), Patients with unstable cervical spine, Pregnant patients, Patients with a full stomach, Patients with severely restricted mouth opening where the introduction of laryngoscope blade is not possible A pre-operative evaluation of the airways to be done by the primary investigator. This will include predictors of difficult airways, Patients posted for emergency surgeries, Patients requiring rapid sequence intubation, Patients on anticoagulants, or having coagulopathy, The patient was kept fasting for 8 hours. On surgery day following changing the patient to O.T, intravenous access was held. Preoperative baseline parameters of heart rate, blood pressure and oxygen saturation were documented and the crystalloid infusion was started. Patient was premedicated with midazolam $1 \mathrm{mg}$ iv slowly; glycopyrrolate $0.2 \mathrm{mg}$ i.v; and ondansetron $4 \mathrm{mg}$ i.v. Inj. fentanyl $2 \mu \mathrm{g} / \mathrm{kg}$ i.v was given over 30 seconds after preoxygenation with $100 \%$ oxygen for 5 minutes utilizing a face mask connected to Bain's circuit. Inj. propofol 2 $\mathrm{mg} / \mathrm{kg}$ was provided for the induction of anaesthesia. This was followed by Inj. succinylcholine $1.5 \mathrm{mg} / \mathrm{kg}$ and pulmonary ventilation were done for 30 seconds. The patient's head was positioned in an impartial position and laryngoscopy was completed with either Truview PCD or Macintosh blade as randomly allocated. Truview PCD blade was commencing from the center of the mouth and respited on the tongue. The endotracheal tube was accumulated on the preformed curved steel styled endow with the laryngoscope. The tube was launched from the angle of the mouth with the concavity facing laterally and was advanced blindly till its tip come into the Truview PCD visual field. Afterward, the tube was turned $90^{\circ}$ and initiated during the vocal cords as seen through the lens.
The Macintosh blade was knob in the standard manner. The "sniffing" position was used. It was placed in as of the right side of the mouth and to the right of the tongue. The laryngoscope was advanced and simultaneously moved into the midline to relocate the tongue to the left. Once the vocal cords were visualized the endotracheal tube was negotiated during the vocal cords.

If the first intubation is unsuccessful, anaesthesiologist was educated to act according to their priority. Optimization maneuvers required if any were taken note of. This included head and neck manipulation, external laryngeal manipulation. The number of efforts until the tube is in position was documented and also instances of altering the laryngoscope blade for an additional size or form of a blade. Time to intubate was noted in both groups. The anaesthesiologist assessed the complexity of performing the intubation. The laryngoscopic view was graded using Cormack and Lehane grading and note was taken. The trachea was intubated with a suitable size endotracheal tube and anaesthesia was sustained. Heart rate, blood pressure and hemoglobin oxygen saturation were documented all through intubation and subsequently. After surgery, the patient was assessed for signs of sore throat. The frequency of broken teeth, soft-tissue edema, bleeding from gums or lips, stridor or hoarseness was recorded also. Data were collected regarding age, gender, weight, pre-anesthesia airway assessment, intubation parameters, and post-extubation signs.

\section{Statistical analysis:}

Sample size calculation was done considering the study by M. Barak et al in 2007 where the meantime to intubation with Truview and Macintosh was 33 seconds and 24seconds respectively and the standard deviation for each was 12 and 13 respectively. for $90 \%$ power of the study, the $Z$ Beta value is 1.282 and for type 1 error of $5 \%$ the $Z$ Alpha value is 1.96 .

Replacing these in the following formula,

$\mathrm{N}=2($ Z Alpha + Z Beta) 2 x s.d. 2

D2

Where s.d. $=$ standard deviation $=13$

$\mathrm{D}=$ difference in mean $=33-24=9$

We got a sample size of 45 patients in each group.

This implies a sample size of 90 ASA I and II patients. They were randomly assigned to laryngoscopy by either Truview PCD laryngoscope or Macintosh laryngoscope by simple randomization using a computer-generated simple randomization table. Randomization was done by a senior faculty in the department.

\section{Results:}


Table 1: Demographic and airway assessment data

\begin{tabular}{|llll}
\hline Variables & $\begin{array}{l}\text { Group 1 Truview blade } \\
(\mathbf{n = 4 5})\end{array}$ & $\begin{array}{l}\text { Group 2 Macintosh } \\
\text { blade }(\mathbf{n = 4 5})\end{array}$ & P-value \\
\hline Age; years & $34.11(15.5)^{*}$ & $35.13(14.3)^{*}$ & 0.37 \\
\hline Weight; kg & $59.44(7.7)^{*}$ & $60.68(10.7)^{*}$ & 0.26 \\
\hline Sex; F:M & $28: 17$ & $26: 19$ & 0.66 \\
\hline ASA grade 1\2 & $35 / 10$ & $36 / 9$ & 0.79 \\
\hline Mouth opening Normal/restricted & $45 / 0$ & $45 / 0$ & $\mathrm{NS}$ \\
\hline Thyromental distance Normal/abnormal & $45 / 0$ & $45 / 0$ & $\mathrm{NS}$ \\
\hline Dental status: Normal /loose/edentulous & $43 / 2 / 0$ & $43 / 2 / 0$ & $\mathrm{NS}$ \\
\hline TMJ mobility Normal / restricted & $45 / 0$ & $45 / 0$ & $\mathrm{NS}$ \\
\hline Mandibular size Normal / micrognathia & $45 / 0$ & $45 / 0$ & $\mathrm{NS}$ \\
\hline Mallampati view Class 1/2/3/4 & $39 / 6 / 0 / 0$ & $41 / 4 / 0 / 0$ & 0.52 \\
\hline Expected difficulty No/yes & $45 / 0$ & $45 / 0$ & $\mathrm{NS}$ \\
\hline
\end{tabular}

(* Data represented as a mean and standard deviation; NS - nonsignificant)

Table 2: Intubation variables:

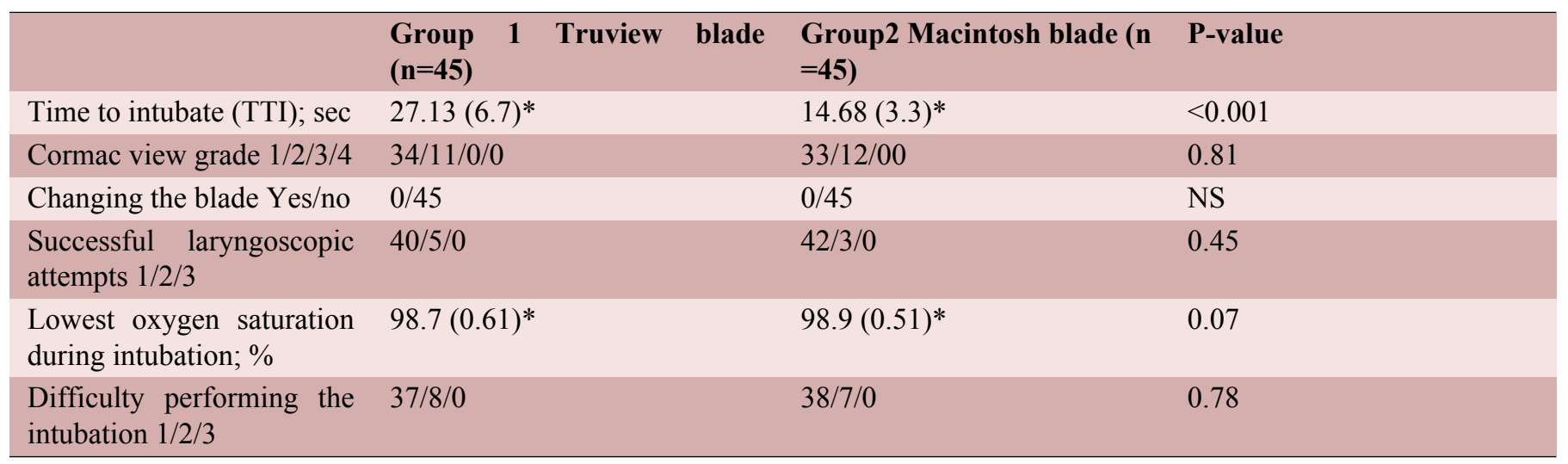

(* Data represented as a mean and standard deviation; NS- nonsignificant)

Table 3: ptimization maneuvers required:

\begin{tabular}{|c|c|c|c|}
\hline & $\begin{array}{l}\text { Group } 1 \text { Truview bla de } \\
(n=45)\end{array}$ & $\begin{array}{l}\text { Group } 2 \text { Macintosh blade } \\
(n=45)\end{array}$ & P-value \\
\hline Head and neck manipulation Yes/no & $8 / 37$ & $7 / 38$ & 0.78 \\
\hline Both yes/both no & $8 / 35$ & $6 / 29$ & 0.87 \\
\hline
\end{tabular}

Discussion:

Truview PCD laryngoscope is a freshly launched appliance with an incorporated optical lens system and distinctive blade tip angulation that offers the finest angle of image permitting a vision of the glottis by the prismatic lens devoid of containing to ally oral, pharyngeal and tracheal axes. ${ }^{[5]}$

In the present research, we have determined the applicability of the Truview laryngoscope in operating rooms as a habit tackle in place of the Macintosh laryngoscope.

Ninety ASA 1 and 2 patients aged 18-60 yrs planned to experience possible surgery under general anaesthesia were incorporated for the research. The mean age of patients in our study was $34.1+15.5$ years and $35.1+14.3$ years in the Truview PCD and Macintosh group respectively. The weight of the patients ranged from 42 to $80 \mathrm{~kg}$ with an average of 59.44 kgs and $60.68 \mathrm{kgs}$ in the Truview PCD and Macintosh group respectively. 


\begin{tabular}{|c|c|c|c|}
\hline Complications & $\begin{array}{l}\text { Group } 1 \\
\text { blade }(n=45)\end{array}$ & $\begin{array}{l}\text { Group } 2 \text { Macintosh blade } \\
(n=45)\end{array}$ & P-value \\
\hline Damaged tooth Yes/no & $0 / 45$ & $0 / 45$ & NS \\
\hline Soft tissue damage Yes/no & $0 / 45$ & $4 / 41$ & 0.12 \\
\hline Bleeding gums/lips Yes/no & $0 / 45$ & $0 / 45$ & NS \\
\hline Sore throat Yes/no & $10 / 35$ & $5 / 40$ & 0.13 \\
\hline Stridor Yes/no & $0 / 45$ & $0 / 45$ & NS \\
\hline Hoarsness Yes/no & $0 / 45$ & $1 / 44$ & 0.5 \\
\hline
\end{tabular}

*NS- Non-Significant

Preoperative airway assessment done in sitting position showed a thyromental distance ranging from a minimum of $6 \mathrm{~cm}$ to a maximum of $11 \mathrm{~cm}$. We did not include any case of abnormal thyromental distance. Interincisor distance varied from $3 \mathrm{~cm}$ to $6 \mathrm{~cm}$, patients with restricted mouth opening were excluded. In the Truview group, $86.67 \%$ of patients had Mallampati class I, $13.33 \%$ of patients had Mallampati class II. In the Macintosh group, $91 \%$ of patients had Mallampati class I, $9 \%$ of patients had Mallampati class II. This was compared using Fisher's exact test and was found to be statistically insignificant. There was no patient with Mallampati class III and IV in our study. Two patients of each group had loose dentures, which remained undamaged during the laryngoscopy. All of the patients had normal cervical mobility and normal temporomandibular joint mobility. There was no patient with micrognathia or receding mandible which predicted difficult airways. Hence there was no patient with expected difficult airways as assessed during the preoperative period. ${ }^{[6]}$

In our study, all of the patients were successfully intubated with Truview or Macintosh laryngoscope. The mean Time to intubate required with the Truview PCD laryngoscope was 27.13 seconds with an SD of 6.7. The mean time to intubate required for Macintosh laryngoscope was 14.68 Seconds with an SD of 3.3. This was statistically significant as compared with students unpaired t-test with a p-value of $<0.0001$.

$33(73.3 \%)$ patients had a Cormack Lehane grade 1 view with Macintosh laryngoscope compared to 34 (75.5\%) patients with a truview PCD laryngoscope. While 12 (26.7\%) patients had grade 2, Cormack and Lehane, with Macintosh laryngoscope, $11(24.5 \%)$ patients had grade 2 view with Truview laryngoscope. There was no definitive improvement in the vocal cord view with the Truview laryngoscope when compared to Macintosh laryngoscope which was statistically tested using the chi-square test. There did not arise any need to change the blade with each the Truview PCD video laryngoscope or the Macintosh laryngoscope. ${ }^{[7,8]}$

Forty (88.9\%) patients Of the Truview group were intubated in the first attempt. Five patients of the Truview group were intubated in the second attempt. Amongst these five patients, during the laryngoscopy of three patients misting of optical viewport occurred which prolonged the intubation time marginally. In the Macintosh group, forty-two (93.33\%) patients were intubated in the first effort and three patients required a second effort. This was compared using Fishers exact test and was found to be statistically non-significant.

There was no clinical or statistical significance in the lowest oxygen saturation recorded during the process of intubation with the Truview PCD laryngoscope (lowest spo2 mean 98.7\%) and the Macintosh laryngoscope (lowest spo2 mean $98.9 \%)$.

Regarding ease of intubation, it was considered to be easy in $37(82.2 \%)$ patients, intermediate in $8(17.8 \%)$ patients in the Truview group. As against this, in the Macintosh group, intubation was considered to be easy in $38(84.4 \%)$ patients and intermediate in $7(15.6 \%)$ patients. This is clinically as well as statistically insignificant when compared using the chisquare test and keeping $\mathrm{p}<0.05$.

In the Truview PCD group, $8(17.78 \%)$ patients required head and neck manipulation which was comparable with $7(15.56 \%)$ patients in the Macintosh group. The no. of patients who required external laryngeal manipulation was 10 $(22.22 \%)$ in the Truview PCD group and $15(33.33 \%)$ in the Macintosh group. This may seem clinically significant but was found to be statistically insignificant at $p<0.05$. In the Truview PCD group, 8 patients required both optimization maneuvers as against 6 patients in the Macintosh group which was statistically non-significant. No patient had soft tissue trauma in the Truview PCD group while $4(8.88 \%)$ patients in the Macintosh group had. Postoperatively, sore throat was observed in $10(22.22 \%)$ patients in the Truview group and 5 (11.11\%) patients in the Macintosh group. This was found to be statistically insignificant.

One of the patients intubated with Macintosh laryngoscope had hoarseness of voice immediately post-extubation which continued in the post-operative period for few hours and resolved thereafter. 
Throughout the trial we did not observe any tooth damage or bleeding gums and lips with either the Truview PCD or the Macintosh laryngoscope.

Even though our results did not demonstrate specific development in the laryngeal view as compared to the Macintosh blade, this may be ascribed to the fact that patients with expected difficult airways were excluded from the study and the Truview laryngoscope significantly improves the laryngeal view in patients with higher Mallampati grading.

The mean Time to intubate required with the Truview PCD laryngoscope was 27.13 seconds with an SD of 6.7. The mean time to intubate required for Macintosh laryngoscope was 14.68 Seconds with an SD of 3.3. This was statistically significant as compared with students unpaired t-test with a p-value of $<0.0001$.

The Truview blade is intended to allow indirect laryngoscopic view; thus, the anaesthetists apply fewer forces on the anterior larynx, ensuing in a smaller number of patients with bleeding and soft tissue damage. ${ }^{[9,10]}$ Through the study, we did not examine any airway trauma with the Truview PCD laryngoscope.

\section{Conclusion}

Truview PCD can be considered an alternate intubation device, especially in difficult intubation conditions.

\section{References}

1. Khan RM, Kaul N, Gupte S, Hinai FSAA. Truview $\mathrm{PCD}^{\mathrm{TM}}$ eases laryngoscopy and tracheal intubation in patient with cleft lip, palate, and protruding premaxilla. Paediatr Anaesth. 2011;21(12):1279-1281. Available from: https://dx.doi.org/10. 1111/j.1460-9592.2011.03684.x.

2. Apfelbaum JL, Hagberg CA, Caplan RA, Blitt CD, Connis RT. Practice guidelines for management of the difficult airway: An updated report by the American Society of Anesthesiologists Task Force on Management of the Difficult Airway. Anesthesiology. 2013;118:251-8209.

3. Torun AÇ, Tür A, Özkan F, Kelsaka E, Karakaya D, Sarıhasan B, et al. Assesment of Macintosh Laryngoscope and Truview EVO2 Video-laryngoscope With Respect to Hemodynamic and Intubation Quality in Patients With Presumptive Difficult
Intubation. J Exp Clin Med. 2011;28(1):4-7. Available from: https://dx.doi.org/10.5835/jecm.omu.28.1.009.

4. Singh I, Khaund A, Gupta A. Evaluation of True view EVO2 laryngoscope in anticipated difficult intubation \&\#8209; A comparison to Macintosh laryngoscope. Indian J Anaesth. 2009;53(2):164-8209.

5. Rocke DA, Murray WB, Rout CC, Gouws E. Relative Risk Analysis of Factors Associated with Difficult Intubation in Obstetric Anesthesia. Anesthesiol. 1992;77:67-73. Available from: https://dx.doi.org/10.1097/00000542-19920700000010 .

6. Caplan RA, Posner KL, Ward RJ, Cheney FW. Adverse Respiratory Events in Anesthesia: A Closed Claims Analysis. Anesthesiol. 1990;72(5):828-833. Available from: https://dx. doi.org/10.1097/00000542-199005000-00010.

7. Cheney FW. The American Society of Anesthesiologists Closed Claims Project: what have we learned, how has it affected practice, and how will it affect practice in the future? Anesthesiol. 1999;91:552-558. Available from: https://doi.org/ 10.1097/00000542-199908000-00030.

8. Peterson GN, Domino KB, Caplan RA, Posner KL, Lee LA, Cheney FW. Management of the Difficult Airway. Anesthesiol. 2005;103(1):33-39. Available from: https://dx.doi.org/10. 1097/00000542-200507000-00009.

9. Williamson JA, Webb RK, Szekely S, Gillies ERN, Dreosti AV. Difficult Intubation: An Analysis of 2000 Incident Reports. Anaesth Intensive Care Med. 1993;21(5):602-607. Available from: https: //dx.doi.org/10.1177/0310057x9302100518.

10. Crisis Management Manual: cover abcd a swift check. Adelaide: Australian Patient Safety Foundation. 1996;p. 74-74.

Copyright: (C) the author(s), 2020. It is an open-access article distributed under the terms of the Creative Commons Attribution License (CC BY 4.0), which permits authors to retain ownership of the copyright for their content, and allow anyone to download, reuse, reprint, modify, distribute and/or copy the content as long as the original authors and source are cited.

How to cite this article: Agrawal N, Shah A, Joshi B, Vasani P. Evaluation of the True View PCD Video Laryngoscope for Oral Endotracheal Intubation. Acad. Anesthesiol. Int. 2020;5(2): $57-61$.

DOI: dx.doi.org/10.21276/aan.2020.5.2.12

Source of Support: Nil, Conflict of Interest: None declared. 